\title{
Response Analysis of thin-walled Structure under Non-uniform temperature field and Acoustic Loads
}

\author{
Yundong Sha, Xiyang Zheng \\ Liaoning Key Laboratory of Advanced Test Technology for Aeronautical propulsion System, \\ Shenyang, 110136, China
}

Keywords: interpolation. On-uniform thermal loads; Acoustic loads; Thin-walled structure; nonlinear dynamic response.

\begin{abstract}
With the raised performance of advanced aircraft, hypersonic vehicles are subjected to increasingly severe environment. Very severe elevated temperature and high intensity acoustic loads will cause the thin-walled structures of aircraft to respond in a strong nonlinear large deflection vibration, and leading to premature fatigue failure of the structure. According to the Von Karman large deflection theory, the large deflection governing equation of plate under thermo-acoustic loadings is obtained. The Galerkin method is employed to obtain the ordinary differential equation under modal coordinates and the stress expressed by modal displacement for the simply-supported plate and the clamped plate. The critical thermal buckling temperatures and modal frequencies of simply-supported plate and thin-walled cylindrical shell under thermal loads with linear temperature gradient, the dynamic responses of them under thermo-acoustic loads, are obtained using finite element numerical stimulation, The thermal-acoustic responses of simply-supported plate and thinwalled cylindrical shell with different combinations of thermal-acoustic loadings are obtained, including the random vibration in pre-buckled regime, snap-through motion between post-buckled equilibrium positions and nonlinear vibration around one post-buckled position.
\end{abstract}

\section{Introduction}

The sonic loads on aircraft are as high as $180 \mathrm{~dB}$, while the temperature could reach $3000{ }^{\circ} \mathrm{f}$. Around the initial equilibrium position, large amplitude nonlinear vibration on the pre-buckling structure can be caused by strong acoustic loads, which makes the buckling structure beat severely between different buckling equilibrium position, namely snap-through, or vibrate around a buckling equilibrium position [1-3]. In order to achieve high speed, aerospace is designed by reducing its weight, its structural parts are often used of thin-walled structure, such as an airplane wing, skin, etc. To improve the flight performance, the thin-walled structure which can show the complex nonlinear response of large deflection is more widely adopted. If the thin-walled structure connects to the support parts which can act as a cooling device, due to the heat transfer effect, there will be thermal load in the form of temperature gradient distribution on the structure. In consequence, it is one of the important issues which aircraft structure design at home and abroad to consider that dynamic response investigation of thin-walled structural under non-uniform thermal loads and acoustic loads [4].

A finite element equation is presented for the prediction of nonlinear responses of curved panels under combined thermo-acoustic loadings. The non-uniform temperature field is considered, and band limited Asian white noise is chosen as random acoustic loading. Thermal buckling temperature and thermal buckling deflections are obtained to explain the snap-through phenomenon. The modal frequency of the vibration about the thermally buckled equilibrium position is studied, which shows great difference between the primary and secondary buckled equilibrium position of curved panels. Displacement and stress response obtained show nonlinear characteristics of curved panels under thermo-acoustic loadings.

\section{Finite Element Formulation}

The system equations of motion in structural node DOF is[5] 


$$
M\{\ddot{w}\}+\left[K-K_{T}+K 1(w)+K 2(w, w)\right]\{w\}=\{P\}+\left\{P_{T}\right\}
$$

where $M$ is the mass matrix, $K$ is the linear stiffness matrix, $w$ is the time dependent vector of nodal displacements and $\{\mathrm{P}\}$ is the time dependent vector of nodal forces. K1and $\mathrm{K} 2$ are nonlinear stiffness matrices which are linear and quadratic functions of the nodal displacements, respectively. $\mathrm{KT}$ is the linear stress stiffness matrix due to induced thermal stresses and $\{\mathrm{PT}\}$ is the vector of nodal forces due to the thermal stress.

A finite element formulation for thermal post-buckling and free vibration of curved panels subjected to temperature variations over the panel is presented in this section.

By neglecting the inertial term and random loading from Eq. 1, the system equations of motion become a set of nonlinear algebraic equation (nonlinear static problem) as [6]

$$
\left[K-K_{T}+K 1(w)+K 2(w, w)\right]\{w\}=\left\{P_{T}\right\}
$$

Newton-Raphson iteration can be used to solve the nonlinear algebraic equation ,the static thermal post-buckling deflection $\{\mathrm{w}\}$ will be not determined until a displacement convergence criterion is satisfied. Once the static thermal post-buckling deflection is obtained for a certain temperature variation over the curved panel, by adding the inertial terms and neglecting the nonlinear stiffness matrices, the linear equation of motion about this thermal post-buckling equilibrium position $\{w\}$ is [6]

$$
M\{\ddot{w}\}_{t}+K_{\tan }\{w\}_{t}=0
$$

Where $\{\mathrm{w}\} \mathrm{t}$ is the dynamic displacement and Ktan is tangent stiffness. The Eq. 3 is then solved, as a basic eigenvalue problem, to obtain the modal frequencies and vibration modes of the thermally buckled curved panel.

$$
\Omega=\left[\omega_{1}, \omega_{2}, \ldots, \omega_{n}\right], \quad \Phi=\left[\phi_{1}, \phi_{2}, \ldots, \phi_{n}\right]
$$

In order to easily understand the problem, Eq. 1 can be equivalent to[7]

$$
M\{\ddot{w}\}+F_{N L}(w, T)=\{P\}
$$

where the overall stiffness term of structure under non-uniform thermal loads and acoustic loads, containing linear stiffness, thermal stress, stiffness, geometric nonlinear stiffness and nonlinear stiffness caused by temperature change. According to equation $\mathrm{W}=\Phi$ displacement of the physical coordinates can be converted to displacement of modal coordinates, then Eq. 1 of the physical coordinates can be converted to equation of modal coordinates as follows.

$$
\tilde{M} \ddot{q}(t)+f_{N L}\left(q_{1}(t), q_{2}(t), \ldots, q_{n}(t), T\right)=p(t)
$$

Where $\tilde{M}=\Phi^{T} M \Phi=I \quad f_{N L}=\Phi^{T} F_{N L} p=\Phi^{T} P$ nonlinear stiffness restoring force term as follows

$$
\begin{aligned}
& f_{N L}^{r}\left(q_{1}, \ldots, q_{n}, T\right) \\
& =\sum_{j=1}^{n} d(T) q_{j}+\sum_{j=1}^{n} \sum_{k=j}^{n} a_{j k}^{r}(T) q_{j} q_{k}+\sum_{j=1}^{n} \sum_{k=j}^{n} \sum_{l=k}^{n} b_{j k l}^{r}(T) q_{j} q_{k} q_{l}, \quad r=1, \ldots, n
\end{aligned}
$$

Where d, a and b represent respectively linear, cubic no linear and quadratic line modal stiffness coefficient. Taking advantage of Newton-Raphson iterative method, nonlinear static equation is solved, then take the results into the equation of modal coordinates to calculate. Using Newark integral method in time-domain, equations are integrated in every specified time step to get modal displacement in time series, then converted to displacement of the physical coordinates, as displacement response of thin-walled structure. Accordingly, taking advantage of relation of stress and strain[8], vibration stress response of thin-walled structure under thermo-acoustic loadings is received.

\section{Numerical Results and Discussions}

\section{Dimensions and Material Properties.}

In this paper, as examples of structures undergoing non-uniform temperature and random acoustic loadings, the rectangular GJB493-88 titanium alloy plate and the curved panel with simplysupported boundary are considered with the following dimensions and material properties, as shown in Table 1. 


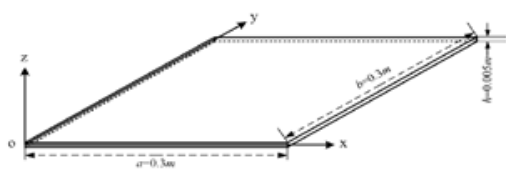

(a) The alloy plate

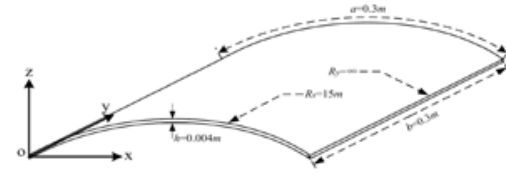

(b) the curved panel

Fig 1. The dimensions of the computing model

Table 1. Material properties of GJB493-88 titanium alloy

\begin{tabular}{cccccc}
\hline Type & $0{ }^{\circ} \mathrm{C}$ & $100{ }^{\circ} \mathrm{C}$ & $200^{\circ} \mathrm{C}$ & $300^{\circ} \mathrm{C}$ & $400^{\circ} \mathrm{C}$ \\
\hline $\mathrm{E}[\mathrm{GPa}]$ & 113 & 109 & 106 & 101 & 97 \\
$\mu$ & 0.3 & 0.3 & 0.3 & 0.3 & 0.3 \\
$\alpha[\mathrm{K}-1]$ & $8.5 \times 10-6$ & $8.5 \times 10-6$ & $8.9 \times 10-6$ & $9.3 \times 10-6$ & $9.6 \times 10-6$ \\
$\rho[\mathrm{Kg} / \mathrm{m} 3]$ & 4370 & 4370 & 4370 & 4370 & 4370 \\
\hline
\end{tabular}

\section{Thermo-acoustic Loadings.}

Considering the alloy plate without inner heat source, the three-dimensional heat conduction differential equation can be transferred to a one dimension heat conduction differential equation of the problem, as follows

$$
d^{2} T / d x^{2}=0
$$

According to the temperature boundary conditions $T_{1}=T(0, y)=\left.T\right|_{x=0} T_{2}=T(a, y)=\left.T\right|_{x=a}$ integrating twice with the both sides of equation, the temperature distribution function for rectangular thin-wall plate is

$$
T(x)=\Delta T(x / a)+T_{1}
$$

where $\Delta T=T_{2}-T_{1}$, if $T_{1}=27.4^{\circ} \mathrm{C}, T_{2}=57.4^{\circ} \mathrm{C}$, the temperature distribution function for the rectangular thin-wall plate is[9]

$$
T(x)=30(x / 0.3)+27.4
$$

Taking advantage of finite element method, make steady state conduction analysis for rectangular thin-wall plate, the temperature gradient distribution along one side of the thin-wall plate edges to the other one can be illustrated clearly in Fig 2.

It is assumed that the reference temperature Tref $=0^{\circ} \mathrm{C}$, the temperature is considered constant through the thickness of the plate, a steady state thermal loading with non-uniform temperature distribution is considered [10].

$$
\Delta T(x, y)=T \sin \left(\frac{\pi x}{l}\right) \sin \left(\frac{\pi y}{w}\right), 0 \leq x \leq l, 0 \leq y \leq w
$$

Where $\mathrm{T}$ is the peak temperature of $\Delta \mathrm{T}(\mathrm{x}, \mathrm{y}), \mathrm{l}$ and $\mathrm{w}$ are length and width of a rectangle flat plate.

$$
\Delta T(x, y)=T \sin \left(\frac{\pi x}{2 \mathrm{R} \sin (a / 2 \mathrm{R})}\right) \sin \left(\frac{\pi y}{b}\right), 0 \leq x \leq 30 \sin \left(\frac{a}{2 \mathrm{R}}\right), 0 \leq y \leq b
$$

A typical simulated non-uniform temperature distribution plot of the curved panel is presented in Fig. 2, and the temperature gradient distribution along the curved panel edges to the heated center can be illustrated clearly in Fig 3.

Uniformly distributed band limited Gaussian white noise is chosen as the random acoustic loading, which is shown in Fig 4.

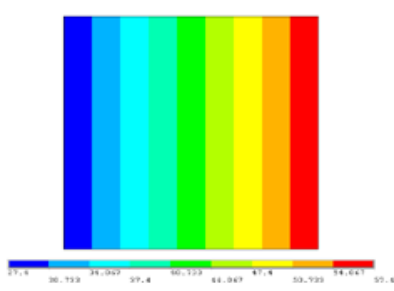

Fig 2. Liner temperature

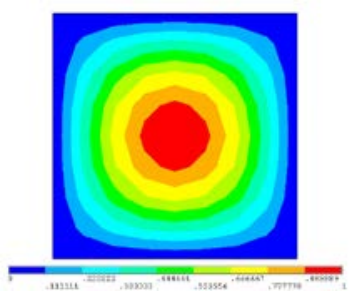

Fig 3. Non-uniform

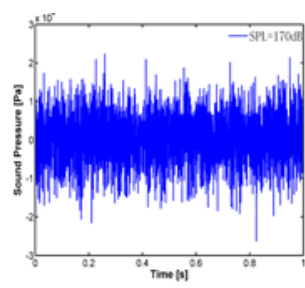

Fig 4. Noise sound pressure 


\section{Thermal buckling analysis and modal analysis.}

The first 6 order critical thermal buckling temperatures of the simply supported curved panel under thermal load with temperature gradient are listed in Table 1 . The coefficient of thermal buckling $\mathrm{S}$ is applied to denote peak temperature, where $\mathrm{S}=\mathrm{T} / \mathrm{Tcr}$, and $\mathrm{T}$ and $\mathrm{Tcr}$ are the actual peak temperature and the first order critical thermal buckling temperature, respectively. At thermal buckling coefficient $\mathrm{S}>1$, the simply supported curved panel under thermal load with temperature gradient will be buckled.

Table 2. Critical thermal buckling temperatures

\begin{tabular}{ccccccc}
\hline Order & 1 & 2 & 3 & 4 & 5 & 6 \\
\hline Tcr $/\left[{ }^{\circ} \mathrm{C}\right]$ & 60.3 & 155.8 & 159.5 & 296.4 & 340.7 & 367.8 \\
\hline
\end{tabular}

Thermal deflection on the initial equilibrium position and the primary branch buckled equilibrium position (BEP) increases positively right away as peak temperature increases form $\mathrm{S}=0$. It is noticed that the secondary branch can be determined until the peak temperature $\mathrm{T}$ reach the critical thermal buckling temperature $\operatorname{Tcr}(S=1)$, and thermal deflection on the secondary branch increases negatively as the peak temperature $\mathrm{T}$ increases from $\mathrm{Tcr}(\mathrm{S}=1)$. It is natural to speculate that random vibration characteristics of the curved panels would have snap-through motions between the primary and secondary buckled equilibrium position after the peak temperature exceeds the critical thermal buckling temperature Tcr. As shown in Fig 5.

There are two independent variables in the linear temperature gradient Eq. 8, namely the temperature difference $\Delta T$, and the boundary heating temperature $T_{1}$. For an example of $\triangle$ $T=30^{\circ} \mathrm{C}$, when $T_{1}=27.4^{\circ} \mathrm{C}$, thermal buckling occurs on the simply supported plate in Fig 6 .

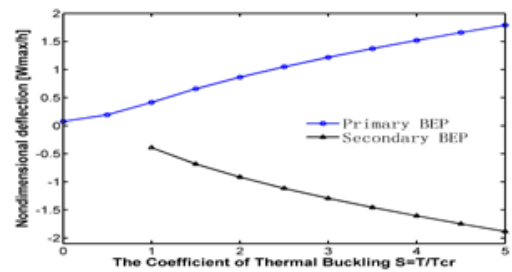

Fig 5. Thermal deflection

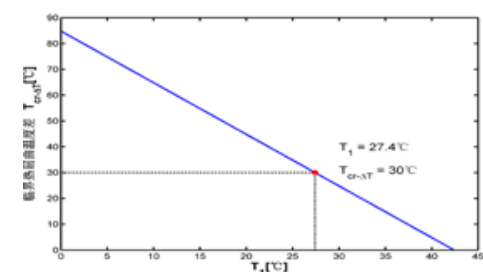

Fig 6. Buckling temperature difference

Table 3. The modal frequencies of the curved panel

\begin{tabular}{cccccccc}
\hline \multirow{2}{*}{$S$} & \multirow{2}{*}{$\left.T /{ }^{\circ} \mathrm{C}\right]$} & \multirow{2}{*}{ Wmax $/ \mathrm{h}$} & \multicolumn{5}{c}{ Modal frequencies $/[\mathrm{Hz}]$} \\
\cline { 4 - 7 } & & & 1 & 2 & 3 & 4 & 5 \\
\hline 0 & 0 & 0 & 234.0 & 571.2 & 573.9 & 909.6 & 1255.0 \\
0.4 & 24.1 & 0.08 & 215.9 & 538.9 & 539.6 & 879.6 & 1218.9 \\
\hline \multirow{2}{*}{1.4} & \multirow{2}{*}{84.4} & 0.52 & 307.4 & 527.3 & 534.7 & 857.1 & 1179.5 \\
& & -0.53 & 264.6 & 501.1 & 511.9 & 838.2 & 1162.2 \\
\hline \multirow{2}{*}{2} & \multirow{2}{*}{120.6} & 0.86 & 381.5 & 546.6 & 557.3 & 861.9 & 1175.5 \\
& & -0.92 & 351.4 & 524.3 & 539.0 & 844.1 & 1160.2 \\
\hline
\end{tabular}

\section{Thermo-acoustic Response Results and Discussion}

At a relative small peak temperature $S=0.4\left(24.1^{\circ} \mathrm{C}\right)$, the dynamic displacement response of the central node in the curved panel at different Sound Pressure Level (SPL) are presented in Fig. 7(a), Fig. 7(b), Fig. 7(c) and Fig. 7(d). It is shown that the curved panel vibrates around the initial equilibrium position, because thermal buckling phenomenon of the curved panel has't been occurred. And the amplitude of the displacement of the curved panel increases gradually with the growth of the SPL.

At a peak temperature $\mathrm{S}=1.4\left(84.4^{\circ} \mathrm{C}\right)$, the random response of the displacement of the central node in the curved panel at different Sound Pressure Level (SPL) are presented in Fig. 8. Because of the peak temperature $S=1.4>1$, the curved panel has come into thermal buckling state, and the curved panel vibrates around the primary or secondary buckled equilibrium position, or vibrates between the primary and secondary buckled equilibrium position. Fig. 8(a), Fig. 8(b) and Fig. 8(c) 
show that the amplitude of the displacement of the curved panel increases gradually as the SPL increases from $140 \mathrm{~dB}$ to $160 \mathrm{~dB}$, and the curved panel vibrates around the primary buckled equilibrium position. At SPL=170dB, the curved panel have come into being snap-through motions between the primary and secondary buckled equilibrium positions, although the main major part of the displacement time history is on the positive side. It is obvious that the primary buckled equilibrium holds dominant position in post-buckling region. It is also noted that the secondary buckled equilibrium position will be appeared only when the acoustic load reaches sufficiently large.

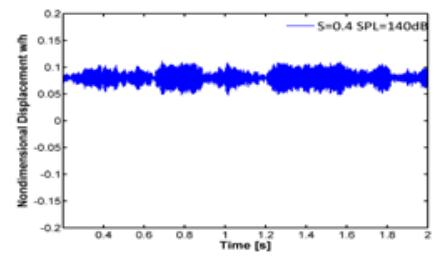

(a) $(\mathrm{S}=0.4, \mathrm{SPL}=140 \mathrm{~dB})$

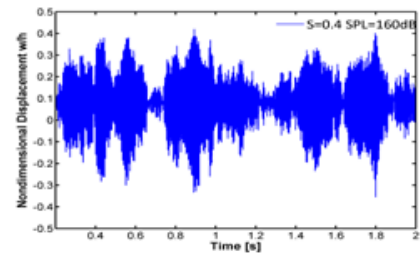

(c) $(\mathrm{S}=0.4, \mathrm{SPL}=160 \mathrm{~dB})$

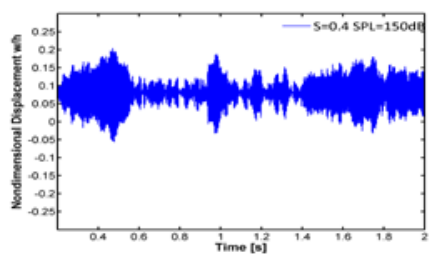

(b) $(\mathrm{S}=0.4, \mathrm{SPL}=150 \mathrm{~dB})$

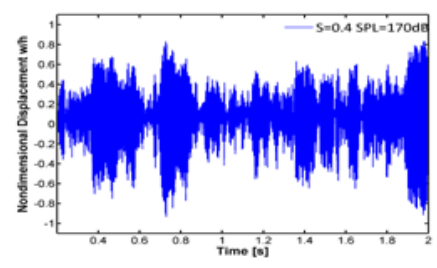

(d) $(S=0.4, S P L=170 \mathrm{~dB})$

Fig. 7 Nonlinear dynamic displacement response of the curved panel

The above results indicate that the response of the curved panel becomes dominated by the amplitude caused by the acoustic loading. It is shown that the random response of the curved panel subjected to random acoustic load and thermal load with non-uniform temperature distribution belongs to the nonlinear large deflection characteristics.

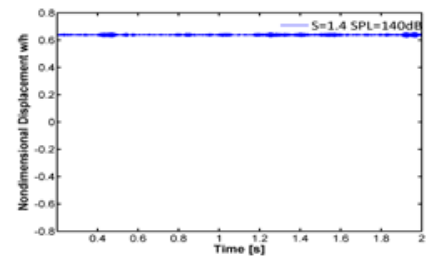

(a) $(\mathrm{S}=1.4, \mathrm{SPL}=140 \mathrm{~dB})$

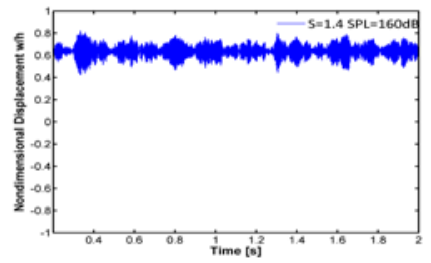

(c) $(\mathrm{S}=1.4, \mathrm{SPL}=160 \mathrm{~dB})$

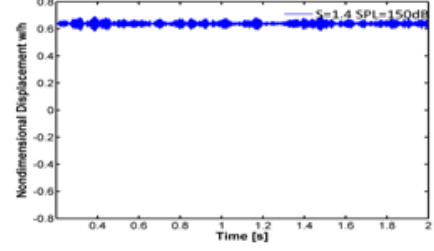

(b) $(S=1.4, S P L=150 \mathrm{~dB})$

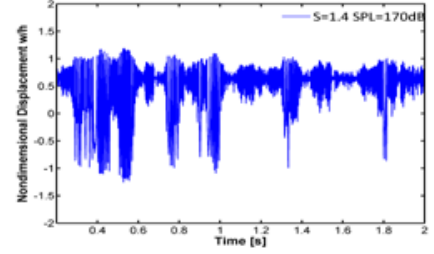

(d) $(S=1.4, \mathrm{SPL}=170 \mathrm{~dB})$

Fig. 8 Nonlinear dynamic displacement response of the curved panel

\section{Conclusions}

(1) Thermal buckling critical temperature of simply-supported thin walled plate under linear temperature gradient loading is the difference between double thermal buckling critical temperature and the minimum temperature on the boundary under uniform temperature loading. When the temperature difference is a constant value, before the thermal buckling, the modal frequency of the plate reduces as the minimum temperature on the boundary increases, when the modal frequency increases as the minimum temperature on the boundary increases, which is consistent with the 
change trend of the modal frequency going with the minimum temperature under uniform temperature loading.

(2) Under non-uniform temperature filed, the primary and secondary buckled equilibrium positions and the fundamental frequencies of the curved panel are identified. It is shown that the frequencies about the secondary buckled equilibrium position is lower than the primary buckled equilibrium position , the results show that it is less stiffness than the primary buckled equilibrium position.

(3) The displacement responses of the curved panel show that the primary buckled equilibrium holds dominant position in post-buckling region. The secondary buckled equilibrium position will be appeared only when the acoustic load reaches sufficiently large. Especially while the curved panel comes into being snap-through motions between the primary and secondary buckled equilibrium positions, the main major part of the displacement time history is on the positive side.

\section{References}

[1] Sha Y D, WeiJ, GaoZ J, et al. Nonlinear Characteristics of Thin-walled Structures under Thermo-acoustic Loadings[J]. Acta Aeronautica et Astronautica Sinica, 2013, 34(006): 1336-1346.

[2] Guo X P, Sha Y D, Bai S S, et al. Application study of the random sonic acoustic fatigue based on the rain-flow counting and power spectrum density[J]. Journal of Aeroengine, 2010, 36(5): 27-31.

[3] Sha Y D, Guo X P, Liao L F, et al. Probability distribution of von Mises stress for complex thinwalled structures undergoing random acoustic loadings[J]. Journal of Vibration and Shock, 2011, 30(1): 137-141.

[4] Sha Y D, ZhuLin, Luan X C,et al.Dynamic response of thin plates under thermal loadings with temperature gradient and acoustic loadings[J].Journal of Vibration and Shock, 2014, 33(18): 102109.

[5] Przekop A., and Rizzi, S.A.Dynamic Snap-Through of Thin Walled Structures by a Reduced Order Method[C], AIAA-2006-1745, 2006.

[6] Przekop A, Guo X, Azzouz M S, et al. Reinvestigation of nonlinear random response of shallow shells using finite element modal formulation[C] Structural Dynamics and Materials Conference. 2004.

[7] Rizzi S A, Przekop A. System identification-guided basis selection for reduced-order nonlinear response analysis[J]. Journal of Sound and Vibration, 2008, 315(3): 467-485.

[8] Dhainaut J M, Guo X, Mei C, et al. Nonlinear random response of panels in an elevated thermalacoustic environment[J]. Journal of aircraft, 2003, 40(4): 683-691.

[9] R. Javaheri, M. R. Eslami. Thermal Buckling of Functionally Graded Plate[J].Javaheri And Eslam AIAA JOURNAL, 2002

[10] Ko W L. Thermal Buckling Analysis of Rectangular Panels Subjected to Humped Temperature Profile Heating[R]. NASA/TP-2004-212041, 2004. 